\title{
Association of Triglyceride Glucose (TyG) Index With Leukoaraiosis in Middle-aged and Older Adults: A Cross-sectional Study
}

Dong-Hyuk Jung

Yonsei University College of Medicine

Byoung Jin Park

Yonsei University College of Medicine

Yong-Jae Lee ( $\sim$ ukyjhome@yuhs.ac)

Yonsei University College of Medicine https://orcid.org/0000-0002-6697-476X

\section{Research}

Keywords: TyG index, leukoaraiosis, white matter disease

Posted Date: December 11th, 2020

DOl: https://doi.org/10.21203/rs.3.rs-124306/v1

License: (c) (i) This work is licensed under a Creative Commons Attribution 4.0 International License.

Read Full License 


\section{Abstract}

Background: Leukoaraiosis refers to lesions of high signal intensity in the periventricular and subcortical white matter that result from chronic microvascular ischemic damage to the brain. Increasing evidence suggests that the triglyceride glucose (TyG) index is associated with arterial stiffness and cardiovascular disease, which are both closely related to ischemic arterial damage. We hypothesized that the serum TyG index could be associated with cerebrovascular microangiopathy as measured by leukoaraiosis among middle-aged and older adults.

Methods: This cross-sectional study included 2,162 Korean adults aged $\geq 45$ years who participated in a health examination program between 2010 and 2011. TyG index was calculated as Ln (fasting triglycerides $(\mathrm{mg} / \mathrm{dl}) \times$ fasting blood glucose $(\mathrm{mg} / \mathrm{dl}) / 2)$. TyG index quartiles were categorized as follows: $\mathrm{Q} 1, \leq 8.12 ; \mathrm{Q} 2,8.13-8.50 \mathrm{Q} 2,8.51-8.89$; and Q4, $\geq 8.90$. The odds ratios (ORs) and 95\% confidence intervals ( $95 \% \mathrm{Cls}$ ) for leukoaraiosis based on brain MRI scans were calculated across TyG index quartiles using multiple logistic regression analysis.

Results: The overall prevalence of leukoaraiosis was $5.4 \%$ and increased with serum TyG index quartiles. Compared to the lowest quartile, the ORs $(95 \% \mathrm{Cls})$ of the highest TyG index quartile for leukoaraiosis was 2.37 (1.17-4.79) after adjusting for age, sex, body mass index, smoking status, alcohol intake, regular exercise, mean arterial pressure, total cholesterol, HDL cholesterol levels, hypertension, and type 2 diabetes.

Conclusions: The serum TyG index was positively and independently associated with leukoaraiosis. Our findings indicate that the TyG index might be a useful additional measure for assessing cerebrovascular microangiopathy in clinical settings.

\section{Background}

The term leukoaraiosis refers to lesions of high signal intensity in the periventricular and subcortical white matter on a fluid-attenuated inversion recovery (FLAIR) or T2-weighted sequence of a magnetic resonance imaging (MRI) scan of the brain. Leukoaraiosis is often found incidentally in asymptomatic, older individuals[1], and the prevalence of leukoaraiosis varies widely from $5.3 \%$ to $95 \%$ depending on age range, ethnicity, comorbidities, and sample size of the study populations[2,3]. It has long been considered to have little clinical significance despite its high prevalence, especially among older people[3]. However, recent studies have suggested that leukoaraiosis may seem asymptomatic but is not a benign physiological aging phenomenon. It has been confirmed to be associated with an increased risk of a wide range of geriatric syndromes such as ischemic stroke, brain atrophy, cognitive impairment, depressive mood, and gait disturbance[4-9]. Although the mechanism of leukoaraiosis development has not been fully elucidated, ischemic cerebral arteriolar damage has been suggested as a central underlying cause[10].Recently, the triglyceride glucose (TyG) index has emerged as a novel marker for atherosclerotic cardiovascular diseases (CVD) and metabolic disorders[11-19]. The TyG index is calculated as Ln (fasting 
triglycerides $(\mathrm{mg} / \mathrm{dl}) \times$ fasting blood glucose $(\mathrm{mg} / \mathrm{dl}) / 2)$. Thus, a high TyG index means hyperglycemic and dyslipidemic status, which play crucial roles in the development and progression of atherosclerosis. It has been reported that a higher TyG index was positively associated with a major adverse cardiovascular event, ischemic stroke, coronary calcification, arterial stiffness, and nephrotic microvascular damage[11-16]. Moreover, an elevated TyG index was significantly associated with a higher risk of insulin resistance, type 2 diabetes, and metabolic syndrome[17-19]. Given the previous findings between TyG index and cardiovascular and metabolic disorders, individuals with a higher TyG index could be more susceptible to cerebrovascular atherosclerosis, but data on the association between them are limited. Therefore, we hypothesized that TyG index could be associated with cerebrovascular microangiopathy measured by leukoaraiosis among middle-aged and older adults.

\section{Methods}

\section{Study participants}

This study is based on the health risk assessment study that aims to characterize CVD risk factors and to explore surrogate markers for CVD in Korean adults. The study cohort consisted of 9,451 individuals aged $\geq 20$ years who voluntarily visited the Health Promotion Center, Gangnam Severance Hospital, Yonsei University College of Medicine for regular health check-ups between March 2010 and May 2011. The current analysis was limited to 2,584 participants aged $\geq 45$ years who received MRI scans. We excluded participants who met at least one of the following criteria: missing data, did not fast for $12 \mathrm{~h}$ prior to testing, or a history of cancer, ischemic heart disease, or stroke $(n=422)$. Following these exclusions, 2,162 participants were included in the final analysis. This study was conducted in accordance with the ethical principles of the Declaration of Helsinki and was approved by the Institutional Review Board of Yonsei University College of Medicine, Seoul, Korea.

\section{Data collection}

Each participant completed a questionnaire about lifestyle and medical history. Self-reported cigarette smoking, alcohol consumption, and physical activity characteristics were gleaned from the questionnaires. The smoking status was categorized as non-smoker, ex-smoker, and current smoker. Questions regarding alcohol intake included the frequency of consumption on a weekly basis. Regular alcohol consumption was defined as alcohol drinking $\geq$ two times per week. Participants were asked about their physical exercise on a weekly basis, and regular exercise was defined as exercise $\geq$ three times per week. Body weight and height were measured to the nearest $0.1 \mathrm{~kg}$ and $0.1 \mathrm{~cm}$, respectively, in lightweight indoor clothing without shoes. Body mass index (BMI) was calculated as the weight in kilograms divided by the square of the height in meters $\left(\mathrm{kg} / \mathrm{m}^{2}\right)$. Systolic blood pressure (SBP) and diastolic blood pressure (DBP) were measured using the patient's right arm with a standard mercury sphygmomanometer in the sitting position after 10 min of rest (Baumanometer, W.A. Baum Co Inc., Copiague, NY, USA). All blood samples were obtained from the antecubital vein after overnight fasting for $12 \mathrm{~h}$. Fasting plasma glucose, total cholesterol, triglyceride, and high density lipoprotein (HDL) cholesterol 
levels were measured by enzymatic methods using a Hitachi 7600 automated chemistry analyzer (Hitachi Co., Tokyo, Japan), and leukocyte counts were quantified using an automated blood cell counter (ADVIA 120, Bayer, NY, USA).

Hypertension was defined by SBP $\geq 140 \mathrm{mmHg}, \mathrm{DBP} \geq 90 \mathrm{mmHg}$, or the current use of hypertension medication. Type 2 diabetes was defined by a fasting plasma glucose level $\geq 126 \mathrm{mg} / \mathrm{dL}$ or the current use of diabetes medications. The modified National Cholesterol Education Program Adult Treatment Panel III (NCEP-ATP III) was used to define metabolic syndrome. Because waist circumference was not measured, we defined obesity as $B M I \geq 25 \mathrm{~kg} / \mathrm{m}^{2}$, as suggested by the position statement of the American College of Endocrinology[20]. Therefore, metabolic syndrome was defined by the presence of

three or more of the following risk factors: obesity with $\mathrm{BMI} \geq 25 \mathrm{~kg} / \mathrm{m}^{2}$, elevated systolic blood pressure $\geq 130 \mathrm{mmHg}$, elevated diastolic blood pressure $\geq 85 \mathrm{mmHg}$, or the current use of hypertension medication, high fasting plasma glucose $\geq 126 \mathrm{mg} / \mathrm{dL}$ or the current use of diabetes medication, high triglycerides $\geq 150 \mathrm{mg} / \mathrm{dL}$, and low HDL cholesterol ( $<40 \mathrm{mg} / \mathrm{dL}$ for men and $<50 \mathrm{mg} / \mathrm{dL}$ for women). An automatic waveform analyzer (model BP-203RPE; Colin Co., Komaki, Japan) was used to measure brachial-ankle pulse wave velocity (baPWV). This instrument simultaneously recorded blood pressure, phonocardiogram, electrocardiogram, and arterial blood pressure at both the left and right brachial arteries and tibial arteries after 10 min of bed rest. Electrocardiogram electrodes were placed on both wrists, and a microphone for the phonogram was placed on the left edge of the sternum. Pneumonic cuffs were wrapped around both the upper arms and ankles and connected to a plethysmographic sensor to determine the volume pulse waveform. Waveforms for the upper arm (brachial artery) and ankle (tibial artery) were stored for $10 \mathrm{sec}$ sample times with automatic gain analysis and quality adjustment. An oscillometric pressure sensor was attached to the cuffs to measure blood pressure at the four extremities. The baPWVs were recorded using a semiconductor pressure sensor $(1200 \mathrm{~Hz}$ sample acquisition frequency) and calculated using the equation: (La-Lb)/ $\Delta T b a$. La and Lb were defined as the distance from the aortic valve to the elbow and to the ankle, respectively. The distance from the suprasternal notch to the elbow $(\mathrm{La})$ was expressed by $\mathrm{La}=0.2195 \mathrm{x}$ height of subject $(\mathrm{cm})-2.0734$, and the distance from the suprasternal notch to the ankle $(\mathrm{Lb})$ was expressed by $L b=0.8129 \times$ height of subject $(\mathrm{cm})+12.328$. The time interval between the arm and ankle distance $(\Delta \mathrm{Tba})$ was defined as the pulse transit time between the brachial and tibial artery pressure waveforms.

\section{Brain MRI acquisition and leukoaraiosis}

All brain MRI scans were obtained with a 3.0 TMR scanner using a standard head coil (GE Signa VH/l; GE Medical Systems, Milwaukee, WI, USA). All participants were examined after administration of a Gd-DTPA contrast agent. The MRI protocol included the collection of T1- and T2-weighted images and FLAIR images. The current study was based on a retrospective review of the results of brain MRI scans. MRI features were independently evaluated by two experienced radiologists who were unaware of the aims of the study and blind to the laboratory findings. We analyzed the kappa statistics to analyze agreement between the two radiologists because the outcome encompassed ordinal scoring, and the kappa index $(95 \% \mathrm{Cl})$ was $0.859(0.796-0.905)$. Leukoaraiosis was diagnosed when there was hyperintensity on the 
T2-weighted images or FLAIR images without hypointensity on the T1-weighted images in the white matter near the lateral ventricles and subcortical areas.

\section{Statistical analysis}

TyG index was defined as $\mathrm{Ln}$ (fasting triglycerides $(\mathrm{mg} / \mathrm{dl}) \times$ fasting blood glucose $(\mathrm{mg} / \mathrm{dl}) / 2)$. The TyG index quartiles were categorized as follows: Q1: $\leq 8.12$, Q2: 8.13-8.50, Q2: 8.51-8.89, and Q4: $\geq 8.90$. The clinical characteristics of the study population according to serum TyG index quartiles were compared using a one-way analysis of variance (ANOVA) or the Kruskal-Wallis test for continuous variables according to the normality of distributions, and the chi-square test was used for categorical variables. Normal distribution was evaluated with the determination of skewness using the Kolmogorov-Smirnov test. Continuous data are presented as mean (standard deviation, SD) or median (interquartile range, IQR), and categorical data are presented as frequencies. The differences of mean, median and proportion between groups were determined using post hoc analysis of ANOVA, Kruskal-Wallis test and chi-squared test using Bonferroni corrections. The odds ratios for leukoaraiosis were calculated using a multiple logistic regression analysis after adjusting for confounding variables across TyG index quartiles. All analyses were conducted using SAS statistical software (version 9.4; SAS Institute Inc., Cary, NC, USA). All statistical tests were two-sided, and statistical significance was determined at $p$ value $<0.05$. Additionally, the probabilities of leukoaraiosis for TyG index are presented with smooth spline curves using $R$ packages version 3.4.3(Institute for Statistics and Mathematics)

\section{Results}

Table 1 shows the demographic and biochemical characteristics of the study participants according to TyG index quartiles. For lifestyle variables, the proportion of current smokers and alcohol drinking increased with TyG index quartiles. For laboratory variables, the mean or median values of age, BMI, blood pressure, fasting plasma glucose, total cholesterol, triglyceride, baPWV, and leukocyte count increased, whereas HDL cholesterol decreased with increasing TyG index quartiles. The prevalence of hypertension and type 2 diabetes increased in accordance with TyG index quartiles.

Table 2 shows the ORs for leukoaraiosis by to the serum TyG index, obesity, lipid profiles and smoking status. When analyzed in all 2162 subjects, the OR for incident leukoaraiosis in the highest TyG index was 2.81 (95\% Cl 1.44-5.57). However, there was no association between leukoaraiosis and smoking status, lipid profiles and obesity.

Table 3 describes the ORs for leukoaraiosis according to the serum TyG index quartiles. Compared to the lowest quartile, the OR $(95 \% \mathrm{Cl})$ of the highest TyG index quartile for leukoaraiosis was $2.37(95 \% \mathrm{Cl} 1.17$ 4.79) after adjusting for age, sex, body mass index, smoking status, alcohol intake, regular exercise, mean arterial pressure, total cholesterol, HDL cholesterol level, hypertension, and type 2 diabetes.

Figure 1 shows the prevalence of leukoaraiosis according to serum TyG index quartiles. The overall prevalence of leukoaraiosis was $5.4 \%$ and increased with serum TyG index quartiles ( $p$ value $=0.027$ ). We 
also investigated the continuous association between TyG index and the incidence of leukoaraiosis. The odds ratio of leukoaraiosis(with 95\%) for between TyG index are presented with smooth spline curves(Figure 2). This figure shows an increasing trend in the incidence of leukoaraiosis with higher TyG index.

\section{Discussion}

In this cross-sectional study, we found that the TyG index was positively and independently associated with leukoaraiosis after controlling for confounding factors including age, sex, BMI, lifestyle behaviors, and chronic diseases that could influence vascular function. Our findings are in agreement with previous results that indicated TyG index is positively associated with increased risk of macrovascular and microvascular complications including coronary heart disease, ischemic stroke, coronary calcification, arterial stiffness, and nephrotic microvascular damage[11-16]. The exact mechanism by which a higher TyG index confers leukoaraiosis is not well known, but some possible explanations for the association deserve consideration. First, endothelial dysfunction, an important step in the progression of atherosclerosis, could play a crucial role in the pathophysiology of leukoaraiosis. Previous studies have shown evidence of endothelial dysfunction in patients with ischemic microangiopathy and leukoaraiosis[10,14]. Consistent with previous results, arterial stiffness assessed by baPWV was increased with increasing TyG index quartiles in the present study. Second, chronic low-grade inflammation could be another contributor to the association between TyG index and leukoaraiosis. A linear increase in leukocyte counts with increasing TyG index quartiles in the present study supports that TyG index is closely linked to systemic inflammation. Third, insulin resistance may be an additional plausible underlying mechanism to explain the association between TyG index and leukoaraiosis. Insulin resistance plays a central role in the pathogenesis of type 2 diabetes and metabolic syndrome, and the prevalence of leukoaraiosis increased in individuals with type 2 diabetes and metabolic syndrome[21,22]. It has been suggested that insulin resistance may accelerate ischemic white matter lesion formation in patients with type 2 diabetes[23]. Moreover, previous studies have shown that the TyG index is significantly correlated with homeostatic model assessment for insulin resistance (HOMA-IR) and a useful predictor for incident type 2 diabetes[17,18]. Lee et al., in a retrospective cohort study of 2,900 Korean adults with a mean age of 44.3 years, reported that individuals with baseline TyG indexes of 8.8 or more had a 2.5 times higher risk of type 2 diabetes regardless of the presence of generalized obesity[18]. In accordance with the previous findings, the prevalence of metabolic syndrome was increased in accordance with the increasing TyG index quartiles in the present study.

\section{Study Strength And Limitations}

We should acknowledge several limitations in our study to the its interpretation. First, this study used a cross-sectional design, which suggests that caution should be used in causal interpretations. Thus, further large-scale prospective studies with long follow-up periods are warranted to elucidate the cause and effect link between TyG index and leukoaraiosis. Second, because the study participants were 
volunteers visiting for health promotion screenings in a single hospital and appeared to be slightly healthier than most community-based cohorts, the study population may not be representative of the general population. Therefore, this study may have been affected by selection bias. Third, only one measurement of triglyceride and fasting plasma glucose levels from a baseline examination were included in the analyses. Lastly, we used the binary classification for leukoaraiosis without adopting a severity scale because we did not measure the severity at the beginning of this study. Thus, the severity of leukoaraiosis, such as Fazekas scale, was not fully considered in the present study.

\section{Conclusions}

In conclusion, the TyG index was positively and independently associated with leukoaraiosis. Our findings indicate that serum TyG index might be a useful additional measure in assessing cerebrovascular microangiopathy in clinical settings.

\section{Declarations}

Acknowledgments: The authors would like to thank those who underwent medical examinations at the Health Promotion Center of Gangnam Severance Hospital in Seoul, Korea.

Author contributions: All authors have accepted responsibility for the content of this submitted manuscript and approved its submission.

Research funding: None declared.

Availability of data and materials: All data and biological material will be available if needed.

Ethics approval and consent to participate: This study was approved by the Institutional Review Board of Yonsei University College of Medicine, and informed consent was obtained from each participant.

Consent for publication: The authors declare that they have no conflict of interest.

Competing interests: The funding organization(s) played no role in the study design; in the collection, analysis, and interpretation of the data; in the writing of the report; or in the decision to submit the report for publication.

\section{References}

1. Fazekas F, Barkhof F, Wahlund L, Pantoni L, Erkinjuntti T, Scheltens P, Schmidt R (2002) CT and MRI rating of white matter lesions. Cerebrovascular diseases 13 (Suppl. 2):31-36

2. De Leeuw F, de Groot JC, Achten E, Oudkerk M, Ramos L, Heijboer R, Hofman A, Jolles J, Van Gijn J, Breteler M (2001) Prevalence of cerebral white matter lesions in elderly people: a population based magnetic resonance imaging study. The Rotterdam Scan Study. Journal of Neurology, Neurosurgery \& Psychiatry 70 (1):9-14 
3. Wen W, Sachdev PS, Li JJ, Chen X, Anstey KJ (2009) White matter hyperintensities in the forties: their prevalence and topography in an epidemiological sample aged 44-48. Human brain mapping 30 (4):1155-1167

4. Debette S, Markus H (2010) The clinical importance of white matter hyperintensities on brain magnetic resonance imaging: systematic review and meta-analysis. Bmj 341:c3666

5. Wardlaw JM, Doubal F, Armitage P, Chappell F, Carpenter T, Muñoz Maniega S, Farrall A, Sudlow C, Dennis M, Dhillon B (2009) Lacunar stroke is associated with diffuse blood-brain barrier dysfunction. Annals of Neurology: Official Journal of the American Neurological Association and the Child Neurology Society 65 (2):194-202

6. Appelman AP, Vincken KL, Van Der Graaf Y, Vlek AL, Witkamp TD, Mali WP, Geerlings MI (2010) White matter lesions and lacunar infarcts are independently and differently associated with brain atrophy: the SMART-MR study. Cerebrovascular Diseases 29 (1):28-35

7. Longstreth W, Manolio TA, Arnold A, Burke GL, Bryan N, Jungreis CA, Enright PL, O'Leary D, Fried L (1996) Clinical correlates of white matter findings on cranial magnetic resonance imaging of 3301 elderly people: the Cardiovascular Health Study. Stroke 27 (8):1274-1282

8. Teodorczuk A, Firbank MJ, Pantoni L, Poggesi A, Erkinjuntti T, Wallin A, Wahlund L-O, Scheltens P, Waldemar G, Schrotter G (2010) Relationship between baseline white-matter changes and development of late-life depressive symptoms: 3-year results from the LADIS study. Psychological medicine 40 (4):603-610

9. Starr JM, Leaper S, Murray AD, Lemmon H, Staff RT, Deary IJ, Whalley LJ (2003) Brain white matter lesions detected by magnetic resosnance imaging are associated with balance and gait speed. Journal of Neurology, Neurosurgery \& Psychiatry 74 (1):94-98

10. Hassan A, Hunt BJ, O'Sullivan M, Parmar K, Bamford JM, Briley D, Brown MM, Thomas DJ, Markus HS (2003) Markers of endothelial dysfunction in lacunar infarction and ischaemic leukoaraiosis. Brain 126 (2):424-432

11. Luo E, Wang D, Yan G, Qiao Y, Liu B, Hou J, Tang C (2019) High triglyceride-glucose index is associated with poor prognosis in patients with acute ST-elevation myocardial infarction after percutaneous coronary intervention. Cardiovascular diabetology 18 (1):150

12. Shi W, Xing L, Jing L, Tian Y, Yan H, Sun Q, Dai D, Shi L, Liu S (2020) Value of triglyceride-glucose index for the estimation of ischemic stroke risk: insights from a general population. Nutrition, Metabolism and Cardiovascular Diseases 30 (2):245-253

13. Hassan A, Hunt BJ, O'Sullivan M, Bell R, D'Souza R, Jeffery S, Bamford JM, Markus HS (2004) Homocysteine is a risk factor for cerebral small vessel disease, acting via endothelial dysfunction. Brain 127 (1):212-219

14. Park K, Ahn CW, Lee SB, Kang S, Nam JS, Lee BK, Kim JH, Park JS (2019) Elevated TyG Index Predicts Progression of Coronary Artery Calcification. Diabetes care 42 (8):1569-1573

15. Lee SB, Ahn CW, Lee BK, Kang S, Nam JS, You JH, Kim MJ, Kim MK, Park JS (2018) Association between triglyceride glucose index and arterial stiffness in Korean adults. Cardiovascular 
diabetology 17 (1):41

16. Zhao S, Yu S, Chi C, Fan X, Tang J, Ji H, Teliewubai J, Zhang Y, Xu Y (2019) Association between macro-and microvascular damage and the triglyceride glucose index in community-dwelling elderly individuals: the Northern Shanghai Study. Cardiovascular diabetology 18 (1):95

17. Guerrero-Romero F, Simental-Mendía LE, González-Ortiz M, Martínez-Abundis E, Ramos-Zavala MG, Hernández-González SO, Jacques-Camarena O, Rodríguez-Morán M (2010) The product of triglycerides and glucose, a simple measure of insulin sensitivity. Comparison with the euglycemichyperinsulinemic clamp. The Journal of Clinical Endocrinology \& Metabolism 95 (7):3347-3351

18. Lee DY, Lee ES, Kim JH, Park SE, Park C-Y, Oh K-W, Park S-W, Rhee E-J, Lee W-Y (2016) Predictive value of triglyceride glucose index for the risk of incident diabetes: a 4-year retrospective longitudinal study. PLoS One 11 (9)

19. Moon S, Park J-S, Ahn Y (2017) The cut-off values of triglycerides and glucose index for metabolic syndrome in American and Korean adolescents. Journal of Korean medical science 32 (3):427-433

20. Einhorn M, FACP, FACE, Daniel (2003) American College of Endocrinology position statement on the insulin resistance syndrome. Endocrine practice 9 (Supplement 2):5-21

21. Lucatelli P, Montisci R, Sanfilippo R, Sacconi B, Suri JS, Catalano C, Saba L (2016) Is there an association between leukoaraiosis volume and diabetes? Journal of neuroradiology 43 (4):273-279

22. Bokura H, Yamaguchi S, lijima K, Nagai A, Oguro H (2008) Metabolic syndrome is associated with silent ischemic brain lesions. Stroke 39 (5):1607-1609

23. Tamura Y, Araki A (2015) Diabetes mellitus and white matter hyperintensity. Geriatrics \& gerontology international 15:34-42

\section{Tables}

Table 1 Clinical characteristics of the study population according to TyG index quartiles 


\begin{tabular}{|c|c|c|c|c|c|c|}
\hline & \multicolumn{6}{|c|}{ TyG index quartiles } \\
\hline & $\begin{array}{l}\text { Q1 } \\
(\leq 8.12)\end{array}$ & $\begin{array}{l}\text { Q2 (8.13- } \\
8.50)\end{array}$ & $\begin{array}{l}\text { Q3 (8.51- } \\
8.89)\end{array}$ & $\begin{array}{l}\text { Q4 } \\
(\geq 8.90)\end{array}$ & $\begin{array}{l}p \\
\text { value }\end{array}$ & Post hoc \\
\hline $\mathrm{n}$ & 534 & 552 & 533 & 543 & & \\
\hline Age (years) & $\begin{array}{l}55.0 \\
(7.7)\end{array}$ & $56.0(7.6)$ & $56.4(7.5)$ & $55.8(7.2)$ & 0.011 & $b$ \\
\hline Females (\%) & 59.1 & 45.1 & 30.0 & 24.7 & $<0.001$ & $a, b, c, d, e$ \\
\hline $\begin{array}{l}\text { Body mass index } \\
\left(\mathrm{kg} / \mathrm{m}^{2}\right)\end{array}$ & $\begin{array}{l}22.5 \\
(2.7)\end{array}$ & $23.5(2.8)$ & $24.3(2.6)$ & $25.1(2.7)$ & $<0.001$ & $a, b, c, d, e, f$ \\
\hline Systolic BP (mmHg) & $\begin{array}{l}120.6 \\
(16.5)\end{array}$ & $\begin{array}{l}124.7 \\
(17.5)\end{array}$ & $\begin{array}{l}127.8 \\
(18.0)\end{array}$ & $\begin{array}{l}129.0 \\
(16.9)\end{array}$ & $<0.001$ & $a, b, c, d, e, f$ \\
\hline Diastolic BP (mmHg) & $\begin{array}{l}74.1 \\
(9.9)\end{array}$ & $77.2(9.9)$ & $\begin{array}{l}79.5 \\
(10.0)\end{array}$ & $80.5(9.7)$ & $<0.001$ & $a, b, c, d, e$ \\
\hline $\begin{array}{l}\text { Mean arterial BP } \\
(\mathrm{mmHg})\end{array}$ & $\begin{array}{l}89.6 \\
(11.4)\end{array}$ & $\begin{array}{l}93.0 \\
(11.9)\end{array}$ & $\begin{array}{l}95.6 \\
(12.1)\end{array}$ & $\begin{array}{l}96.7 \\
(11.7)\end{array}$ & $<0.001$ & $a, b, c, d, e$ \\
\hline $\begin{array}{l}\text { Fasting plasma glucose } \\
\text { (mg/dL) }\end{array}$ & $\begin{array}{l}87.9 \\
(11.1)\end{array}$ & $\begin{array}{l}95.5 \\
(12.3)\end{array}$ & $\begin{array}{l}101.0 \\
(17.6)\end{array}$ & $\begin{array}{l}112.1 \\
(31.2)\end{array}$ & $<0.001$ & $a, b, c, d, e, f$ \\
\hline $\begin{array}{l}\text { Total cholesterol } \\
(\mathrm{mg} / \mathrm{dL})\end{array}$ & $\begin{array}{l}187.2 \\
(33.6)\end{array}$ & $\begin{array}{l}194.4 \\
(34.3)\end{array}$ & $\begin{array}{l}195.3 \\
(35.4)\end{array}$ & $\begin{array}{l}202.0 \\
(37.2)\end{array}$ & $<0.001$ & $a, b, c, e, f$ \\
\hline Triglyceride $(\mathrm{mg} / \mathrm{dL})$ & $\begin{array}{l}59(51- \\
68)\end{array}$ & $87(78-96)$ & $\begin{array}{l}118(106- \\
133)\end{array}$ & $\begin{array}{l}194(160- \\
242)\end{array}$ & $<0.001$ & $a, b, c, d, e, f$ \\
\hline $\begin{array}{l}\text { HDL-cholesterol } \\
(\mathrm{mg} / \mathrm{dL})\end{array}$ & $\begin{array}{l}59.0 \\
(13.4)\end{array}$ & $\begin{array}{l}52.3 \\
(12.0)\end{array}$ & $\begin{array}{l}47.1 \\
(10.1)\end{array}$ & $43.2(9.6)$ & $<0.001$ & $a, b, c, d, e, f$ \\
\hline $\begin{array}{l}\text { Pulse wave velocity } \\
(\mathrm{cm} / \mathrm{s})\end{array}$ & $\begin{array}{l}1371 \\
(230)\end{array}$ & $\begin{array}{l}1428 \\
(255)\end{array}$ & $\begin{array}{l}1456 \\
(256)\end{array}$ & $\begin{array}{l}1480 \\
(257)\end{array}$ & $<0.001$ & $a, b, c, e$ \\
\hline $\begin{array}{l}\text { Leukocyte count (cells/ } \\
\mu \mathrm{L} \text { ) }\end{array}$ & $\begin{array}{l}5142 \\
(1527)\end{array}$ & $\begin{array}{l}5730 \\
(1605)\end{array}$ & $\begin{array}{l}6041 \\
(1671)\end{array}$ & $\begin{array}{l}6656 \\
(1604)\end{array}$ & $<0.001$ & $a, b, c, d, e, f$ \\
\hline Current smoking (\%) & 9.9 & 15.6 & 21.6 & 33.1 & $<0.001$ & $a, b, c, d, e, f$ \\
\hline Alcohol drinking (\%) & 26.4 & 29.4 & 35.1 & 43.1 & $<0.001$ & $b, c, e$ \\
\hline Regular exercise (\%) & 42.8 & 42.7 & 43.3 & 37.7 & 0.225 & NA \\
\hline Hypertension (\%) & 25.1 & 38.0 & 48.0 & 49.5 & $<0.001$ & $a, b, c, d, e$ \\
\hline Type 2 diabetes (\%) & 3.3 & 6.4 & 11.4 & 24.1 & $<0.001$ & $b, c, d, e, f$ \\
\hline Metabolic syndrome (\%) & 3.0 & 7.1 & 15.7 & 53.4 & $<0.001$ & $a, b, c, d, e, f$ \\
\hline
\end{tabular}

Abbreviations: BP, blood pressure; HDL, high density lipoprotein; NA, not applicable. Data are expressed as the mean (SD), median (IQR), or percentage. P-values were calculated using 1-way ANOVA test, Kruskal- 
Wallis test, or chi-square test. The differences of mean, median and proportion between groups were determined using post hoc analysis of ANOVA, Kruskal-Wallis test and chi-squared test using Bonferroni corrections: a, Q1 versus Q2; b, Q1 versus Q3, c: Q1 versus Q4; d, Q2 versus Q3; e, Q2 versus Q4, and f, Q3 versus Q4. Alcohol drinking $\geq$ twice/week. Regular exercise $\geq$ three times /week.

Table 2. Odds ratios for leukoaraiosis according to baseline TyG index levels in men and women

\begin{tabular}{|ll|}
\hline & Odds Ratios ${ }^{*}(95 \%$ Confidence Interval) \\
\cline { 2 - 2 } Age, years & $1.11(1.08-1.14)$ \\
\hline Sex & 1.00 \\
\hline Men & $1.06(0.96-1.93)$ \\
\hline Women & \\
\hline Body mass index & 1.00 \\
\hline Nonobese $\left(<25 \mathrm{~kg} / \mathrm{m}^{2}\right)$ & $1.06(0.74-1.65)$ \\
\hline Obese $\left(\geq 25 \mathrm{~kg} / \mathrm{m}^{2}\right)$ & \\
\hline TyG index & 1.00 \\
\hline Quartile $1(\leq 8.12)$ & $1.30(1.11-2.42)$ \\
\hline Quartile 2 $(8.13-8.50)$ & $1.11(0.62-2.19)$ \\
\hline Quartile 3 $(8.51-8.89)$ & $2.81(1.44-5.57)$ \\
\hline Quartile 4 ( $\geq 8.90)$ & $1.00(0.99-1.01)$ \\
\hline Smoking & $1.00(0.89-1.00)$ \\
\hline Never smoker & $1.02(1.00-1.04)$ \\
\hline Current smoker & $1.00(0.69-1.17)$ \\
\hline Hypertension & $0.70-1.27)$ \\
\hline Mean arterial pressure & \\
\hline Fasting blood glucose, mg/dL & $1.93-1.02)$ \\
\hline Total cholesterol, mg/dl & \\
\hline HDL-Cholesterol mg/dl & \\
\hline
\end{tabular}


*Adjusted for age, sex, obesity, TyG index, smoking, mean arterial pressure, fasting blood glucose, hypertension, total cholesterol, HDL-cholesterol and TyG index.

Table 3 Odds ratios and 95\% confidence for leukoaraiosis according to TyG index quartiles

\begin{tabular}{|lllll|}
\hline \multicolumn{5}{|l|}{ TyG index quartiles } \\
& Q1 $(\leq 8.12)$ & Q2 (8.13-8.50) & Q3 (8.51-8.89) & Q4 ( $\geq 8.90)$ \\
\hline Model 1 & 1.00 & $1.09(0.55-2.13)$ & $1.20(0.64-2.22)$ & $2.83(1.43-5.55)$ \\
\hline Model 2 & 1.00 & $0.97(0.49-1.92)$ & $1.13(0.60-2.09)$ & $2.52(1.28-4.98)$ \\
\hline Model 3 & 1.00 & $0.94(0.47-1.87)$ & $1.11(0.60-2.06)$ & $2.37(1.17-4.79)$ \\
\hline
\end{tabular}

Model 1: adjusted for age, sex, body mass index, smoking status, alcohol intake, regular exercise, mean arterial pressure, total cholesterol, and HDL-cholesterol levels.

Model 2: adjusted for age, sex, body mass index, smoking status, alcohol intake, regular exercise, mean arterial pressure, total cholesterol, HDL-cholesterol levels, and hypertension.

Model 3: adjusted for age, sex, body mass index, smoking status, alcohol intake, regular exercise, mean arterial pressure, total cholesterol, HDL-cholesterol levels, hypertension, and type 2 diabetes.

\section{Figures}




\section{$\mathrm{P}=0.029$}

( $p$ value was calculated by ANOVA test).

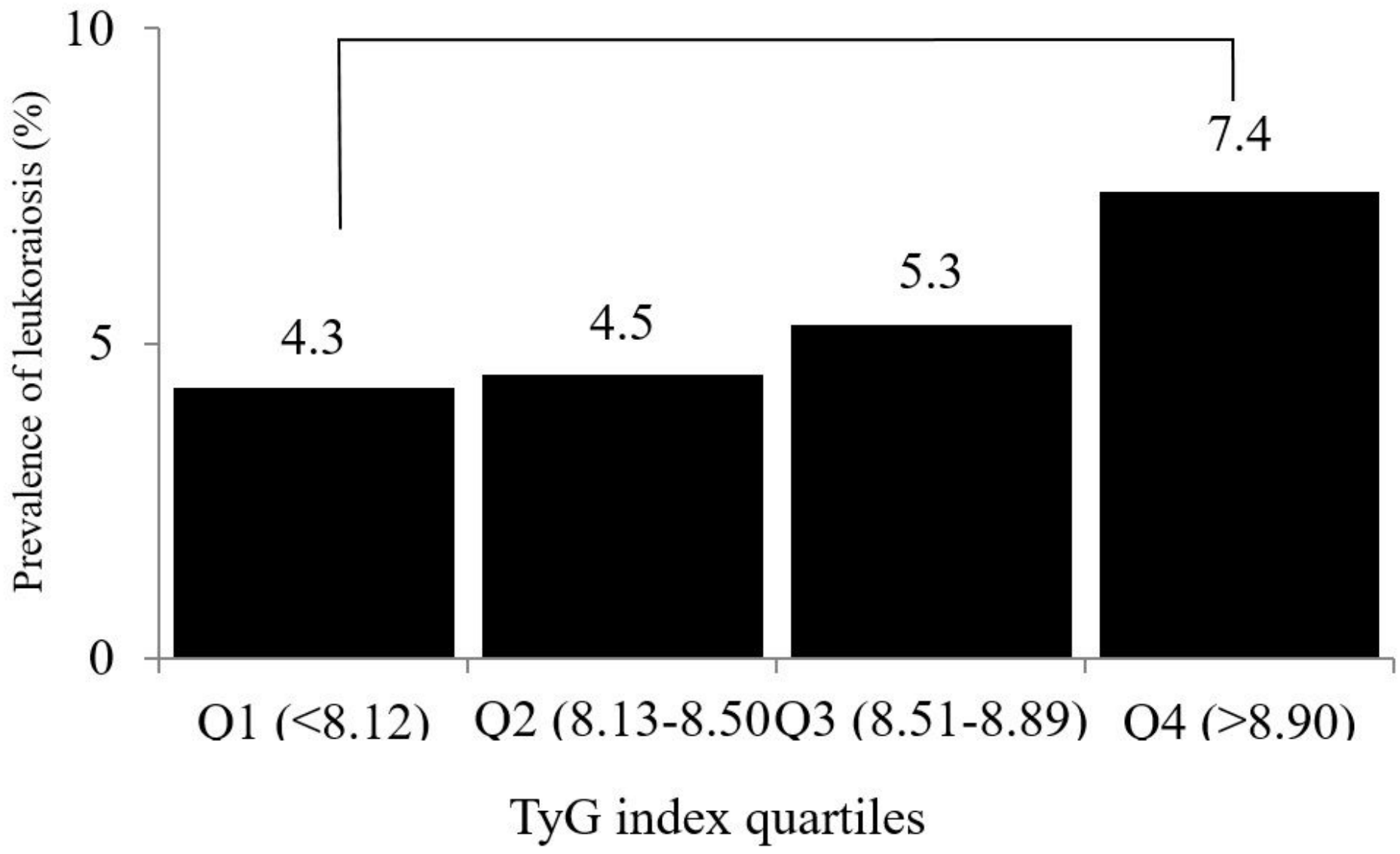

Figure 1

Prevalence of leukoaraiosis according to the triglyceride glucose (TyG) index 


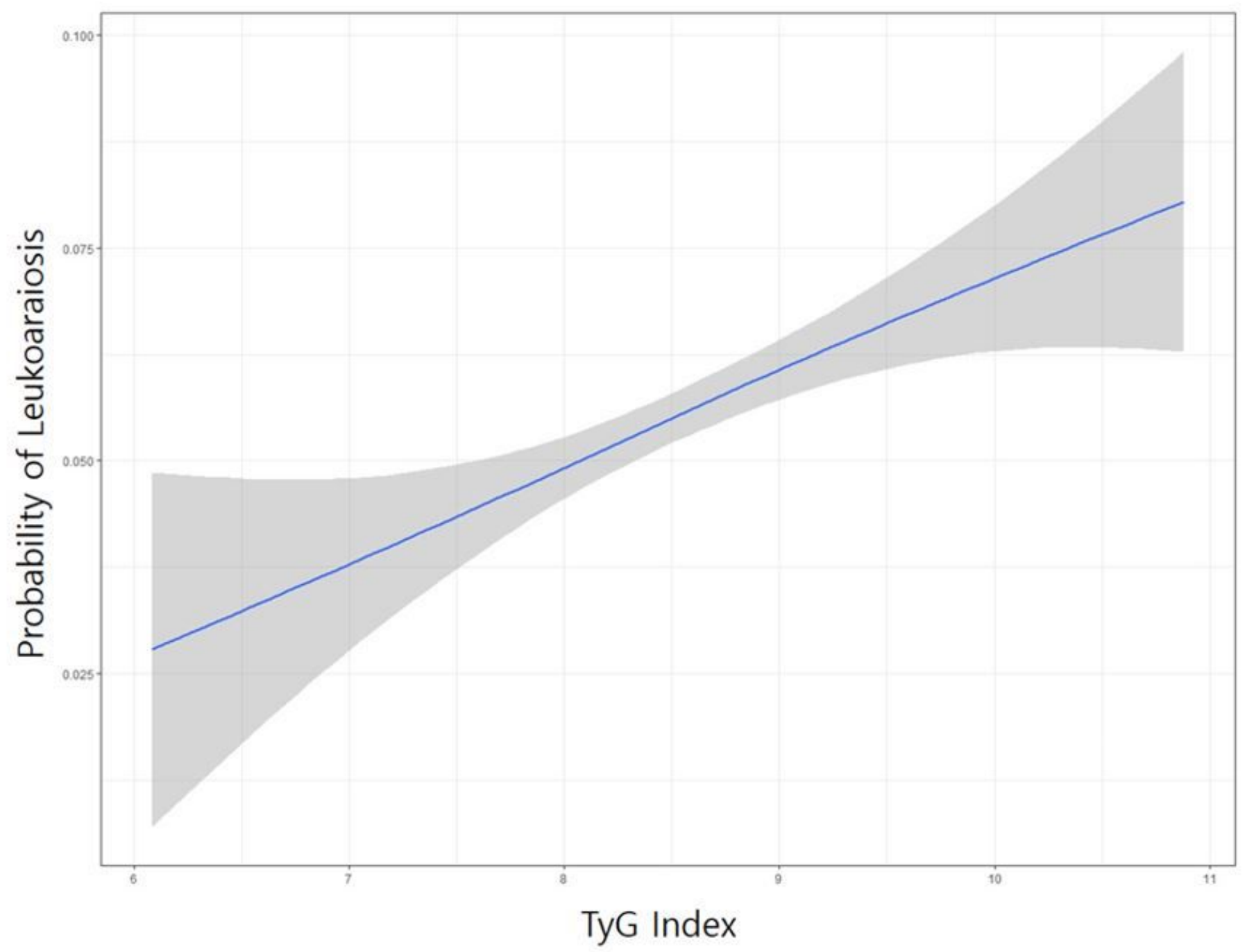

Figure 2

Probability of leukoaraiosis for the triglyceride glucose (TyG) index. Leukoaraiosi s incidence according to TyG index 